\title{
Decisions hint that not all biologics are created with equal ease
}

The Biologics Price Competition and Innovation Act, signed into US law last March, gave the country's drug regulators the authority to establish a pathway to approving 'generic versions' of biologic drugs. Now the US Food and Drug Administration (FDA) must take the next step and set guidelines for evaluating these large, complex organic molecules that are almost-but not quitecopies of some of modern medicine's most important drugs.

As the FDA held a public hearing about the abbreviated pathway for these biosimilars earlier this month, many industry analysts were examining the agency's recent favorable decisions regarding generic versions of the biologic drugs Lovenox (Sanofi Aventis) and Neupogen (Amgen). Although these biosimilar versions might have received an easier pass than many suspected, some industry experts say that the drugs just represent low-hanging fruit.

"In a broader view, they were probably relatively easy choices," says Michael Malecki, head of biosimilar research at Decision Resources, a Burlington, Massachusetts-based company specializing in healthcare analysis.

Simply put, biologic drugs are complex organic molecules that are typically produced by genetically engineered cells. And because a biologic medicine is produced by living cells, it's impossible to say that a generic version using a different cell line is identical. By comparison, small-molecule drugs are easily reproducible because they are made using an invariable chemical process.

In many analysts' views, Lovenox barely qualifies as a biologic. The fast-acting derivative of the blood thinner heparin is made by processing the intestinal mucosal cells of pigs. Although refining the molecule is an extremely complicated process, the drug isn't derived from a genetically modified organism. On the basis of relatively simple clinical trials and chemical characterizations, the FDA green-lighted a biosimilar form of the nearly $\$ 5$ billion drug in July, stating that the generic is entirely interchangeable with the original.

"Despite the simplicity of the drug, a lot of people saw this approval as laying a lot of the groundwork for more sophisticated or 'true' biologics," Malecki says.

\section{True to form}

True biologics are commonly divided into three unofficial categories. The most basic is chemicals that are reproductions of simple

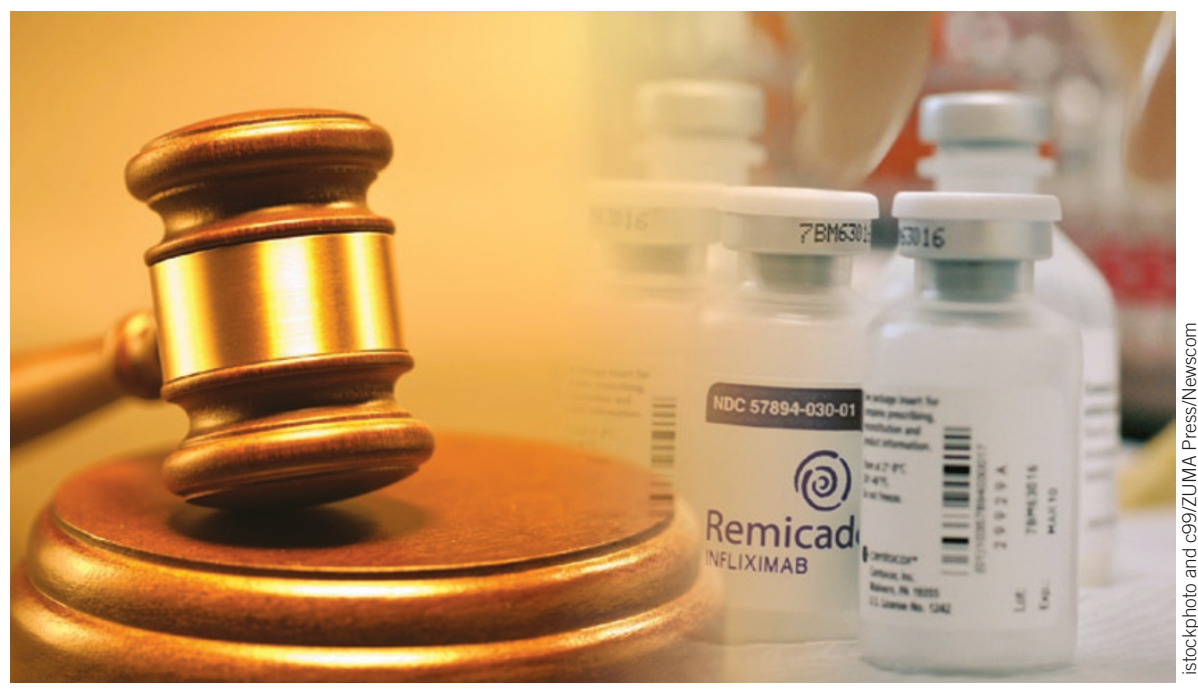

Similar, but different: More complex biologics will be harder to replicate.

human signaling proteins such as human growth hormone and cytokines. These proteins are typically produced in genetically engineered bacteria such as Escherichia coli.

When it comes to making generic versions, "these are the most easily reproduced," says Robin Thorpe, head of the Biotherapeutics Group for the UK's National Institute for Biological Standards and Control, based in Hertfordshire, England.

Neupogen, a hormone used during chemotherapy to stimulate production of white blood cells called neutrophils, falls into this basic category. Teva, the maker of a biosimilar form called Neutroval, submitted an application for approval along with data from five clinical trials-far fewer than a full three-phase drug approval process typically requires. On 30 September, the FDA hinted at a positive response, asking for further information about the drug but not for any additional clinical trials.

"There is only so much you can pull from what the FDA has done, but it's encouraging that the FDA is moving forward in a positive way," Thorpe says. "Europe is paying close attention to what develops and how it deals with some of the trickier questions."

\section{It's complicated}

The EU has had an abbreviated pathway for biologics since 2004 and thus far has approved 14 comparatively simple biosimilars. However, this month the European Medicines Agency is expected to release additional draft guidelines on the second, and generally considered to be more complex, type of biologics: monoclonal antibodies. Officials from the agency have indicated that the guidelines will mandate additional clinical trials for these biologics.

Monoclonal antibodies are slightly modified forms of human antibodies, produced in genetically engineered mammalian cell lines. These include arthritis drugs such as Humira (Abbott) and Remicade (Centocor Ortho Biotech), as well as cancer drugs such as Herceptin (Genentech). Their molecular structures are far more complex than those of hormone biologics. Whereas monoclonal antibodies contain tens of thousands of atoms, hormone biologics possess just a few thousand. Aspirin, by comparison, contains only 21 atoms.

The third and most complex category of biologics is that of fusion proteins, produced from the joining of multiple genes that originally coded for different proteins. These include anemia drug Epogen (Amgen) and the arthritis drug Enbrel (Amgen/Pfizer).

"These are a nightmare to reproduce," Malecki says. It's all the complexity of monoclonal antibodies with additional genetic engineering.

Thus far, the EU has examined biosimilars on a case-by-case basis, requiring additional evidence as the complexity of the drug is increased. Despite efforts to construct additional guidelines, many analysts expect a similar tack to be taken by the FDA.

"There is a basic truth that you really are dealing with a new drug and a new situation in each case," Thorpe says. "There's no avoiding that."

Stu Hutson 